\title{
Thermal buckling and post-buckling of FGM Timoshenko beams on nonlinear elastic foundation
}

\author{
Yun Sun ${ }^{\mathrm{a}, \mathrm{b}}$, Shi-Rong Li ${ }^{\mathrm{a}, \mathrm{b}}$, and Romesh C. Batra ${ }^{\mathrm{c}}$ \\ ${ }^{a}$ School of Civil Science and Engineering, Yangzhou University, Yangzhou, Jiangsu, China; ${ }^{\text {bS }}$ chool of Hydraulic, Energy \\ and Power Engineering, Yangzhou University, Yangzhou, Jiangsu, China; 'Department of Biomedical Engineering and \\ Mechanics, Virginia Polytechnic Institute and State University, Blacksburg, Virginia, USA
}

\begin{abstract}
Buckling and post-buckling thermomechanical deformations of a functionally graded material (FGM) Timoshenko beam resting on a two-parameter nonlinear elastic foundation and subjected to only a temperature rise have been numerically investigated with the shooting method. The material properties are assumed to vary only in the thickness direction according to a power law function. Through-the-thickness temperature distribution is determined by numerically solving the one-dimensional heat conduction equation. Geometric non-linearities in the strain-displacement relations and the non-linear traction-displacement relations at the interface between the beam and the foundation are considered. For clamped-clamped and immovable simply supported beams, critical values of the ratio of temperatures of the top and the bottom surfaces of the beam for transitions in buckling modes to occur are determined. Post-buckled equilibrium paths and configurations of the heated FGM beam are illustrated for different values of the elastic foundation stiffness parameters, exponent in the power law variation of material properties and the slenderness ratio. Results for the Timoshenko beam are compared with those of the corresponding homogeneous Euler-Bernoulli beam available in the literature.
\end{abstract}

\section{ARTICLE HISTORY}

Received 22 January 2015

Accepted 17 February 2015

\section{KEYWORDS}

Buckling mode transition; FGM beam; non-linear elastic foundation; post-buckling equilibrium configurations

\section{Introduction}

Thermal buckling and post-buckling deformations of isotropic homogeneous beams with and without an elastic foundation have been well-investigated in the literature. For example, Li et al. [1-3] presented a mathematical model of the post-buckling deformations of elastic beams under different boundary conditions and subjected to either uniform [1,2] or non-uniform [3] temperature rise. The coupled non-linear ordinary differential equations were numerically solved by the shooting method. The work has been extended to Timoshenko beams $[4,5]$ subjected to thermomechanical loads.

Thermal buckling and post-buckling deformations of Euler-Bernoulli beams (EBBs) resting on twoparameter non-linear elastic foundations were studied by Song and Li [6] and Li and Batra [7] for different boundary conditions. It was found that the critical temperature depended linearly on the linear elastic foundation stiffness parameter. Vaz et al. [8] used the perturbation method to provide an analytical solution for the initial post-buckling behavior of a slender rod supported on a linear elastic foundation and subjected to a uniform temperature gradient.

Functionally graded materials (FGMs) are usually composed of two different homogeneous constituents, such as a ceramic and a metal. The material properties vary continuously in one or more directions, and this variation can be exploited to optimize a functionality of the structure. When FGM structures are used in high-temperature environments, they may buckle under thermal loads. 
However, they may still perform well while being in post-buckled configurations. Therefore, a thorough understanding of thermal buckling and post-bucking deformations of FGM structures, such as FGM beams, is very helpful in understanding their load carrying capacity.

Kiani and Eslami $[9,10]$ analyzed the buckling of FGM beams by using both the EBB and the Timoshenko beam (TB) theories, and gave a closed-form expression for the critical buckling temperature for three types of thermal loads. Ma and Lee [11] obtained a closed-form solution for the non-linear static response of both clamped-clamped and pinned-pinned FGM beams subjected to in-plane thermal loads. Zhao et al. [12] studied post-buckling deformations of immovable simply-supported (S-S) FGM EBBs subjected to a thermal load and presented thermal post-buckling equilibrium paths for different values of the material gradient parameter. The post-buckling of clamped FGM TBs was numerically studied by Li et al. [13] with the shooting method. Based on the non-linear first-order shear deformation beam theory (FSDT), Ma and Lee [14] used the shooting method to numerically study non-linear deformations of S-S FGM beams subjected to uniform in-plane thermal loads. Anandrao et al. [15] investigated the thermal post-buckling behavior of uniform slender FGM beams. They used a single-term Ritz method and the finite element method (FEM) to find the non-linear response of the beams.

There are a few studies on buckling and post-buckling deformations of FGM beams resting on elastic foundations. Sahraee and Saidi [16] applied the differential quadrature method (DQM) to analyze buckling and free vibration of a deep FGM beam-column resting on a Pasternak-type elastic foundation. Fallah and Aghdam $[17,18]$ studied the post-buckling and non-linear free vibrations of FGM EBBs resting on a non-linear elastic foundation subjected to axial compressive [17] and thermomechanical [18] forces. They used a variational method to obtain an approximate closed-form solution of the governing equations. Esfahani et al. [19] analyzed thermal buckling and post-buckling deformations of FGM TBs resting on three-parameter non-linear elastic foundations with five possible boundary conditions in conjunction with two types of thermal loads.

The generalized differential quadrature method (GDQM) was employed to discretize equilibrium equations derived by using the principle of virtual work. Ghiasian et al. [20] studied static and dynamic buckling of an FGM EBB subjected to uniform thermomechanical loads and resting on a threeparameter non-linear elastic foundation with hardening/softening cubic non-linearity. More recently, Esfahani et al. [21] studied small amplitude vibrations of an FGM TB under in-plane thermal loads in the pre-buckling and post-buckling regimes. The GDQM in conjunction with the Newton-Raphson method was used to numerically solve the non-linear governing equations. Shen and Wang [22] investigated non-linear free vibrations, non-linear bending and thermal post-buckling of FGM beams resting on an elastic foundation in a thermal environment. They deduced material properties of the homogenized material by using two micromechanics models, namely, the Voigt and the Mori-Tanaka. Equations of motion were established by using a higher order shear deformation beam theory and incorporating the von Kármán non-linear strain-displacement relations. Praveen and Reddy [23] have studied non-linear transient thermoelastic deformations of a FGM plate.

In the above-mentioned works [17-22] on the post-buckling analysis of FGM beams resting on elastic foundations, geometric non-linearities are taken into account in the sense of von Kármán's straindisplacement relations. It has been pointed out by Batra and Xiao [27] that there is no stress tensor that is work conjugate of the von Kármán strain tensor. They considered all geometric non-linearities that include the von Karman non-linearities. Furthermore, the beam material was assumed to be St. VenantKirchhoff for which the 2nd Piola-Kirchhoff stress tensor is a linear function of the Green-St. Venant strain tensor.

A few investigations have dealt with the influence of an elastic foundation on the equilibrium paths of buckled and post-buckled beams subjected to thermal loads. However, the relation between the critical temperature and the linear elastic foundation stiffness parameters for an FGM beam subjected to nonuniform temperature rise has not been considered in them. In this work, based on the non-linear straindisplacement relations for a TB, the governing equations of a FGM TB resting on a non-linear elastic foundation are derived and numerically solved by the shooting method. Material properties of the FGM beam are assumed to vary in the thickness direction according to a power-law function of the thickness 
coordinate. The through-the-thickness temperature variation governed by the one-dimensional heat conduction equation is computed. The governing equations of the thermal post-buckling deformations are solved via the shooting method to investigate the effect of elastic foundation stiffness parameters on the critical buckling temperature and the equilibrium paths of the beam.

\section{Governing equations}

A schematic sketch of the problem studied is shown in Figure 1. We use rectangular Cartesian coordinates with the $x$-axis originating from the left edge of the beam and located on its mid-surface and the positive $z$-axis pointing upward along the normal to the mid-surface. The beam of rectangular cross-section $b \times h$ and length $l$ rests on a two-parameter non-linear elastic foundation.

\section{Material properties of FGMs}

The FGM beam is assumed to be made of two isotropic constituents. For analyzing the problem, the FGM beam is replaced by a beam made of an equivalent in homogeneous and isotropic material with material properties varying only in the thickness direction. The effective material property, $P$ (i.e., Young's modulus $E$, thermal conductivity $\kappa$, and thermal expansion coefficient $\alpha$ ), are expressed as [23]

$$
P(z)=P_{b} \psi_{P}(z)
$$

where

$$
\psi_{P}(z)=1+\left(P_{r}-1\right)\left(\frac{1}{2}+\frac{z}{h}\right)^{n}
$$

$P_{r}=P_{t} / P_{b}, P_{t}$ and $P_{b}$ denote the value of $P$ at the top $(z=h / 2)$ and the bottom $(z=-h / 2)$ surfaces, respectively, of the beam. However, we assume that Poisson's ratio $\mu$ is a constant which is approximately valid when Poisson's ratios of the two constituent materials are nearly the same. Thus the effective shear modulus $G(z)$ can be expressed as

$$
G(z)=\frac{E(z)}{2(1+\mu)}
$$

Furthermore, the material properties are considered to be independent of the temperature.

\section{Temperature field}

We assume that the temperature varies only in the thickness direction and denote its change from that in the natural or the undeformed state of the beam by $T(z)$. The value of $T(z)$ is found by solving the one-dimensional steady-state heat conduction equation $[13,23]$

$$
\frac{\mathrm{d}}{\mathrm{d} z}\left[\kappa(z) \frac{\mathrm{d} T(z)}{\mathrm{d} z}\right]=0
$$

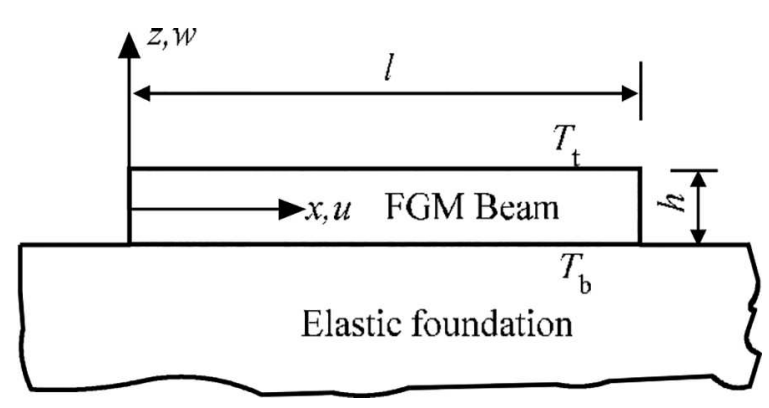

Figure 1. Schematic sketch of the problem studied. 
and the associated boundary conditions

$$
T(h / 2)=T_{t}, T(-h / 2)=T_{b}
$$

where $T_{t}$ and $T_{b}$ denote the temperature change at the top and the bottom surfaces of the beam, respectively. We assume that $T_{b} \neq 0$ and write

$$
T(z)=T_{b} t(z)
$$

in which $t(z)$ is given by

$$
t(z)=1+\left(T_{r}-1\right) \int_{-h / 2}^{z} \frac{\mathrm{d} z}{\kappa(z)} / \int_{-h / 2}^{h / 2} \frac{\mathrm{d} z}{\kappa(z)}
$$

where $T_{r}=T_{t} / T_{b}$. Thus $T_{r}=1$ represents the uniform temperature rise in the beam. Knowing $\kappa(z)>$ 0 , and the temperature at the top and the bottom surfaces of the beam, one can find the temperature at any point in the beam. If $T_{b}=0$ we normalize the temperature by $T_{t}$ in Eq. (6) and suitably modify equation (7).

\section{Strain-displacement relations}

As reported in Refs. [2,7] and [13], the strain-displacement relations can be written as

$$
\frac{\mathrm{d} s_{0}}{\mathrm{~d} x}=\Lambda_{0}, \frac{\mathrm{d} u_{0}}{\mathrm{~d} x}=\Lambda_{0} \cos \theta-1, \frac{\mathrm{d} w_{0}}{\mathrm{~d} x}=\Lambda_{0} \sin \theta
$$

where $s_{0}(x)$ is the arc length of the deformed central axis, $u_{0}(x)$ and $w_{0}(x)$ are displacements of a point on the central axis in the $x$ - and the $z$-directions, respectively, $\Lambda_{0}(x)$ is the stretch at a point on the central axis, and $\theta(x)$ is the angle an element of the deformed central line makes with the $x$-axis.

In the TB theory the transverse shear strain is assumed to be independent of the $z$-coordinate. Thus a cross-section initially perpendicular to the $x$-axis is rotated by the angle

$$
\varphi=\theta+\gamma
$$

about the $y$-axis. The axial strain, $\varepsilon$, and the transverse shear strain, $\gamma^{\prime}$ (not to be confused with $\mathrm{d} \gamma / \mathrm{d}$ $x)$, at the point $(x, z)$ are given by $[4,13]$

$$
\varepsilon=\Lambda_{0} \cos \gamma-1-z \frac{\mathrm{d} \varphi}{\mathrm{d} x}, \gamma^{\prime}=\Lambda_{0} \sin \gamma
$$

\section{Constitutive equations}

In the TB theory, the beam is assumed to undergo plane strain deformations in the $x z$-plane. Furthermore, the beam's thickness is assumed not to change. Thus beam's cross-sectional area remains unaffected by the deformations. However, the cross-section may undergo large rotations. Batra [25] has discussed predictions from four constitutive relations for finite elastic deformations that express a stress tensor as a linear function of the appropriate strain tensor. Allix and Corigliano [26], and Batra and Xiao [27] have used the St. Venant-Kirchhoff material model, respectively, for the TB and the beam with deformations governed by a third-order shear and normal deformable beam theory (TSNDT). For problems studied here, the maximum total axial strain and the maximum angle of rotation of a cross-section are anticipated to be about 0.01 and 0.2 radians, respectively. The total axial strain consists of the thermal strain and the strain produced by the stress. We thus use Hooke's law to first find the horizontal and the vertical forces acting on the undeformed cross-section and then their components along and perpendicular to the beam's deformed axis. 
Using Hooke's law, the stress-strain relations are expressed as

$$
\sigma=E(z)[\varepsilon-\alpha(z) T(z)], \quad \tau=G(z) \gamma^{\prime}
$$

The resultant axial and transverse normal forces, $N$ and $Q$, respectively, and the bending moment, $M$, are defined by

$$
N=\iint_{A} \sigma \mathrm{d} A, \quad M=-\iint_{A} \sigma z \mathrm{~d} A, \quad Q=\frac{1}{k} \iint_{A} \tau \mathrm{d} A
$$

where $k$ is the shear correction factor whose value depends on the shape of the cross-section. Here, $k=3 / 2$ is used for the rectangular section [19].

Substituting for strains from Eq. (10) into Eq. (11), and then for stresses into Eq. (12) results in

$$
\begin{gathered}
N=A_{1}\left(\Lambda_{0} \cos \gamma-1\right)-B_{1} \frac{\mathrm{d} \varphi}{\mathrm{d} x}-N_{T} \\
M=-B_{1}\left(\Lambda_{0} \cos \gamma-1\right)+D_{1} \frac{\mathrm{d} \varphi}{\mathrm{d} x}+M_{T} \\
Q=\frac{1}{k} C_{1} \Lambda_{0} \sin \gamma
\end{gathered}
$$

where $A_{1}, B_{1}, C_{1}$ and $D_{1}$ are stiffness coefficients, and $N_{T}$ and $M_{T}$ are, respectively, the thermal axial force and the thermal bending moment. These are given by

$$
\begin{gathered}
\left(A_{1}, B_{1}, D_{1}\right)=\iint_{A}\left(1, z, z^{2}\right) E(z) \mathrm{d} A=\left(A \phi_{1}, A h \phi_{2}, I \phi_{3}\right) E_{b} \\
C_{1}=\iint_{A} G(z) \mathrm{d} A=\frac{E_{b} A}{2(1+\mu)} \phi_{1} \\
\left(N_{T}, M_{T}\right)=\iint_{A}(1, z) \alpha(z) E(z) T(z) \mathrm{d} A=\left(\phi_{4}, h \phi_{5}\right) N_{T}^{b}
\end{gathered}
$$

where $A$ and $I$ are, respectively, the cross-sectional area and the moment of inertia of the cross-section about the $y$-axis, and $N_{T}^{b}$ is the axial force due to temperature change. These quantities and the dimensionless coefficients $\phi_{i}$ are defined as

$$
\begin{gathered}
A=b h, I=A h^{2} / 12, N_{T}^{b}=\alpha_{b} E_{b} T_{b} A \\
\phi_{1}=\frac{1}{h} \int_{-h / 2}^{h / 2} \psi_{E}(z) \mathrm{d} z, \phi_{2}=\frac{1}{h^{2}} \int_{-h / 2}^{h / 2} \psi_{E}(z) z \mathrm{~d} z, \phi_{3}=\frac{12}{h^{3}} \int_{-h / 2}^{h / 2} \psi_{E}(z) z^{2} \mathrm{~d} z \\
\phi_{4}=\frac{1}{h} \int_{-h / 2}^{h / 2} \psi_{E}(z) \psi_{\alpha}(z) t(z) \mathrm{d} z, \phi_{5}=\frac{1}{h^{2}} \int_{-h / 2}^{h / 2} \psi_{E}(z) \psi_{\alpha}(z) t(z) z \mathrm{~d} z
\end{gathered}
$$

where $\psi_{E}(z)$ and $\psi_{\alpha}(z)$ are given by Eq. (2) with $P=E$ and $\alpha$ for Young's modulus and the thermal expansion coefficient, respectively.

For a homogeneous beam, $\phi_{2}=0$, and $\phi_{1}=\phi_{3}=1$ when $n$ tends to infinity and $\phi_{1}=\phi_{3}=E_{r}$ for $n=0$. Furthermore, for a uniform temperature rise $\left(T_{r}=1\right)$, we have $\phi_{5}=0$, and $\phi_{4}=1$ when $n$ tends to infinity and $\phi_{4}=E_{r} \alpha_{r}$ for $n=0$. 


\section{Equilibrium equations}

Equilibrium equations derived in Refs. [4], [7] and [13] can be written as

$$
\frac{\mathrm{d} H}{\mathrm{~d} x}=\Lambda_{0} q_{x}, \quad \frac{\mathrm{d} V}{\mathrm{~d} x}=\Lambda_{0} q_{z}, \quad \frac{\mathrm{d} M}{\mathrm{~d} x}=\Lambda_{0}(-H \sin \theta+V \cos \theta)
$$

where $H$ and $V$ are the horizontal and the vertical resultant forces, respectively, and $q_{x}$ and $q_{z}$ are distributed loads in the $x$-and the $z$-directions, respectively, exerted by the foundation on the deformed beam.

We assume that the reaction force exerted on the beam by the non-linear elastic foundation can be expressed in terms of the displacements of beam's centroidal axis as [7]

$$
q_{x}=-k_{1} u_{0}-k_{2} u_{0}\left(u_{0}^{2}+w_{0}^{2}\right), \quad q_{z}=-k_{1} w_{0}-k_{2} w_{0}\left(u_{0}^{2}+w_{0}^{2}\right)
$$

where $k_{1}$ and $k_{2}$ can be interpreted, respectively, as the linear and the non-linear foundation stiffness (their units are different); and $u_{0}$ and $w_{0}$, are respectively, displacements of a point on the beam's centroidal axis in the $x$-and the $z$-directions. For non-uniform temperature distribution along the beam thickness, the centroidal axis may not coincide with the neutral axis of the beam.

Resultant forces $N$ and $Q$ are related to $H$ and $V$ by

$$
N=-H \cos \varphi-V \sin \varphi, \quad Q=-H \sin \varphi+V \cos \varphi
$$

Substitution for $N, M$ and $Q$ from Eqs. (13)-(15) into Eq. (20) yields

$$
\begin{gathered}
\frac{\mathrm{d} \varphi}{\mathrm{d} x}=\frac{-\phi_{1} c\left(-M+h \phi_{5} N_{T}^{b}\right)+h \phi_{2} c\left(-H \cos \varphi-V \sin \varphi+\phi_{4} N_{T}^{b}\right)}{\phi_{1} E_{b} I} \\
\Lambda_{0} \sin \gamma=\frac{2 k(1+\mu)(-H \sin \varphi+V \cos \varphi)}{E_{b} A \phi_{1}} \\
\Lambda_{0} \cos \gamma=\frac{-12 \phi_{2} c\left(-M+h \phi_{5} N_{T}^{b}\right)+h \phi_{3} c\left(-H \cos \varphi-V \sin \varphi+\phi_{4} N_{T}^{b}\right)}{E_{b} A h \phi_{1}}+1
\end{gathered}
$$

where $c=1 /\left(\phi_{3}-12 \phi_{2}^{2} / \phi_{1}\right)$ is a dimensionless coefficient. For $c=1$, Eqs. (21)-(23) reduce to those for a homogeneous beam with material properties equal to those of the bottom surface of the FGM beam.

Equations (8), (18) and (21) constitute a system of seven non-linear coupled ordinary differential equations (ODEs) for seven unknown functions, $s_{0}(x), u_{0}(x), w_{0}(x), \varphi(x), H(x), V(x)$ and $M(x)$ defined on the domain $[0, l]$. We note that $\Lambda_{0}$ and $\gamma$ can be expressed in terms of these seven functions.

\section{Equilibrium equations in terms of non-dimensional variables}

We introduce the following non-dimensional variables:

$$
\begin{gathered}
(\xi, S, U, W)=\left(x, s_{0}, u_{0}, w_{0}\right) / l, \quad \delta=l / h, \quad\left(K_{1}, K_{2}\right)=\frac{\left(k_{1}, k_{2} l^{2}\right) l^{4}}{E_{b} I} \\
\left(P_{H}, P_{V}, P_{N}, P_{N T}, m, m_{T}\right)=\frac{\left(H l, V l, N l, N_{T} l, M, M_{T}\right) l}{E_{b} I}, \quad \tau=\frac{N_{T}^{b} l^{2}}{E_{b} I}=12 \alpha_{b} T_{b} \delta^{2}
\end{gathered}
$$

The value of $\tau$ is found by assuming the beam material to be homogeneous with values of material parameters of the material of the bottom surface of the FGM beam and the temperature rise to be uniform; $\tau$ is used as the reference thermal load parameter. 
In terms of the above non-dimensional parameters, Eqs. (8), (18) and (21)-(23) become

$$
\begin{gathered}
\frac{\mathrm{d} S}{\mathrm{~d} \xi}=\Lambda_{0}, \quad \frac{\mathrm{d} U}{\mathrm{~d} \xi}=\Lambda_{0} \cos \theta-1, \quad \frac{\mathrm{d} W}{\mathrm{~d} \xi}=\Lambda_{0} \sin \theta \\
\frac{\mathrm{d} \varphi}{\mathrm{d} \xi}=\frac{c}{\delta \phi_{1}}\left[\phi_{1}\left(\delta m-\phi_{5} \tau\right)-\phi_{2}\left(P_{\mathrm{H}} \cos \varphi+P_{\mathrm{V}} \sin \varphi-\phi_{4} \tau\right)\right] \\
\frac{\mathrm{d} P_{\mathrm{H}}}{\mathrm{d} \xi}=-\Lambda_{0} U\left[K_{1}+K_{2}\left(U^{2}+W^{2}\right)\right] \\
\frac{\mathrm{d} P_{\mathrm{V}}}{\mathrm{d} \xi}=-\Lambda_{0} W\left[K_{1}+K_{2}\left(U^{2}+W^{2}\right)\right] \\
\frac{\mathrm{d} m}{\mathrm{~d} \xi}=\Lambda_{0}\left(-P_{\mathrm{H}} \sin \theta+P_{\mathrm{V}} \cos \theta\right)
\end{gathered}
$$

where

$$
\begin{aligned}
\Lambda_{0} & =\sqrt{s_{1}^{2}+s_{2}^{2}}, \gamma=\arctan \frac{s_{1}}{s_{2}} \\
s_{1} & =\frac{k(1+\mu)}{6 \delta^{2} \phi_{1}}\left(-P_{\mathrm{H}} \sin \varphi+P_{\mathrm{V}} \cos \varphi\right) \\
s_{2} & =\frac{c}{12 \delta^{2} \phi_{1}}\left[12 \phi_{2}\left(\delta m-\phi_{5} \tau\right)-\phi_{3}\left(P_{\mathrm{H}} \cos \varphi+P_{\mathrm{V}} \sin \varphi-\phi_{4} \tau\right)\right]+1
\end{aligned}
$$

From Eq. (29), we conclude that for $\gamma=0$, i.e., $\varphi=\theta, s_{1}=0$, and Eqs. (21)-(29) reduce to those for an EBB.

Boundary conditions for clamped-clamped and immovable simply supported beams are: Clamped edges (C-C):

$$
U(0)=0, W(0)=0, \varphi(0)=0, U(1)=0, W(1)=0, \varphi(1)=0
$$

Immovable simply supported edges (S-S):

$$
U(0)=0, W(0)=0, m(0)=0, U(1)=0, W(1)=0, m(1)=0
$$

For the arc length measured from the left edge, we have $S(0)=0$.

The normalization conditions added to specify a buckled configuration of the FGM beam are: $\varphi(0)=$ $\varphi_{0}$ for an immovable S-S beam, and $m(0)=m_{0}$ for a C - C beam.

\section{Numerical results and discussion}

The FGM beam is assumed to be made of a ceramic (zirconia) and a metal (aluminum) with the top surface made of pure ceramic and the bottom one of pure metal with the following values of material parameters: $E_{t}=E_{c}=151 \mathrm{GPa}, \alpha_{t}=\alpha_{\mathrm{c}}=10 \times 10^{-6} \mathrm{~K}^{-1}, \kappa_{t}=\kappa_{c}=2.09 \mathrm{~W} / \mathrm{m} \cdot \mathrm{K}$ for zirconia; and $E_{b}=E_{m}=70 \mathrm{GPa}, \alpha_{b}=\alpha_{m}=23 \times 10^{-6} \mathrm{~K}^{-1}, \kappa_{b}=\kappa_{m}=204 \mathrm{~W} / \mathrm{m} \cdot \mathrm{K}$ for aluminum. Poisson's ratio for both materials is taken to be 0.3 , i.e., $\mu \equiv 0.3$.

The coupled non-linear ODEs (25)-(28) under the boundary conditions (30) are numerically solved by the shooting method and setting the relative error limit, $E P S=10^{-5}$, for integration using the Runge-Kutta method and the iterative scheme using the Newton-Raphson method. Because differential equations (25)-(28) and boundary conditions (30) are in terms of non-dimensional variables, the three geometric parameters of the beam reduce to only one, the slenderness ratio, $\delta=l / h$. Unless stated otherwise, numerical results have been computed for $\delta=30$. 
The response of the pre-buckled FGM beam is found by setting $W \equiv \theta \equiv 0, \Lambda_{0}=1$ in Eqs. (25)-(28) and (30), which results in $U=0, P_{H}=-P_{N}=\phi_{4} \tau$.

\section{Critical buckling temperature and buckling mode transitions}

By linearizing Eqs. (25)-(28) and (30), i.e., setting $\Lambda_{0}=1, \sin \theta \approx \theta \approx W^{\prime}, \cos \theta=1$, and $P_{H}=\phi_{4} \tau$, and neglecting all non-linear terms in the unknown functions, we get a linear equation for the critical buckling temperature of a FGM beam resting on the elastic foundation; e.g., see Ref. [7]. In the numerical work, it can be found in the limit of $m_{0}$ (or $\varphi_{0}$ ) approaching zero.

To ensure the accuracy of the present method, we have compared in Table 1 the presently computed critical temperature rise, $\Delta T_{c r}$, of C-C FGM TBs subjected to uniform temperature rise without elastic foundation with those given in Ref. [19] based on the GDQM. For the SUS304/Si3N4 FGM beam studied in Ref. [19], $E_{b}=207.8 \mathrm{GPa}, \alpha_{b}=15.3 \times 10^{-6} \mathrm{~K}^{-1}, \kappa_{b}=6.35 \mathrm{Wm}^{-1} \mathrm{~K}^{-1}$ for the metal and $E_{t}=322.3 \mathrm{GPa}, \alpha_{t}=7.47 \times 10^{-6} \mathrm{~K}^{-1}, \kappa_{t}=8.77 \mathrm{Wm}^{-1} \mathrm{~K}^{-1}$ for the ceramic, $\delta=20$, and the shear correction factor, $k=1.2$. It is clear from the values listed in Table 1 that the presently computed values of $\Delta T_{c r}(\mathrm{~K})$ agree well with those reported in Ref. [19].

The critical buckling temperature $\tau_{c r}$ as a function of the linear foundation stiffness parameter $K_{1}$ for the C-C FGM TB is depicted in Figure 2. It is seen that the critical temperature decreases with an increase in the volume fraction index $n$ and the temperature ratio $T_{r}$. For a specified value of $n$, the buckling temperature increases with an increase in the value of $K_{1}$. Results for $K_{1}=0$ are for a beam without an elastic foundation, and those for $K_{1}=0$ and $n=\infty$ are for a homogeneous beam with no elastic foundation. Each critical buckling temperature curve is piecewise linear with line segments of different slopes which correspond to distinct buckling modes. The point of intersection, $A_{m n}$, of two consecutive line segments gives the value of $\left(K_{1}\right)_{m n}$ at which the buckling mode transitions from mode $m$ to mode $n$.

From the results it can be seen that in different ranges of values of the stiffness parameter $K_{1}$ the critical buckling thermal load corresponds to different buckling modes. In other words, for the C-C FGM beam resting on an elastic foundation and hence subjected to follower type (or displacement-dependent)

Table 1. Comparison of $\Delta T_{C r}(\mathrm{~K})$ for C-C Timoshenko FGM beam subjected to uniform temperature rise without elastic foundation, i.e., $K_{1}=K_{2}=0$.

\begin{tabular}{lccccccc}
\hline$n$ & 0 & 0.5 & 1 & 2 & 5 & 10 \\
\hline This work & 693.05 & 510.14 & 458.91 & 423.75 & 394.61 & 376.34 & 338.12 \\
Ref. [19] & 692.70 & 509.89 & 458.68 & 423.53 & 394.39 & 376.14 & 337.94 \\
\hline
\end{tabular}

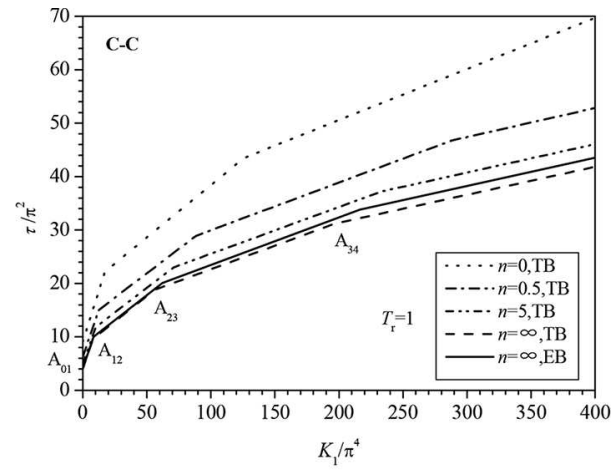

(a) For different values of the exponent $n\left(T_{r}=1\right)$

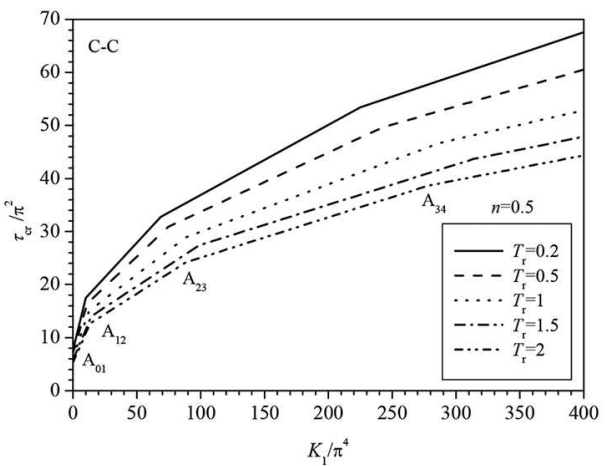

(b) For different values of the temperature ratio $T_{r}(n=0.5)$

Figure 2. Critical temperature for the C-C FGM beam resting on a linear elastic foundation for different values of (a) exponent $n$, and (b) temperature rise ratio $T_{r}$. 
continuously distributed loads the lowest critical temperature need not correspond to the first buckling mode. That is, a higher-order buckling mode could have a lower buckling temperature than that for the immediately lower order buckling mode. The coordinates of the transition points in the buckling modes are listed in Tables 2 and 3 and are compared with the results of Li and Batra [7], and of Wu and Zhong [24] for homogeneous EBBs. Wu and Zhong studied a mechanical problem, thus values of $\tau_{c r}$ for the mode transition are not listed in the table. It is clear that the critical temperature for the homogeneous EBB is a little higher than that for the TB due to shear deformations considered in the TB but not in the EBB. An increase in the value of $T_{r}$ from 0.2 to 1.5 monotonically increases values of $K_{1}$ and $\tau_{c r}$ at which the buckling mode transitions to the next higher one. However, when $T_{r}$ is increased from 1.5 to 2 , values of $K_{1}$ and $\tau_{c r}$ decrease when the buckling mode transitions to the next higher one.

The equilibrium path of the S-S homogeneous beam under uniform or non-uniform temperature rise is not of bifurcation-type. The critical buckling temperatures for a S-S homogeneous TB under uniform temperature rise as a function of the linear foundation stiffness parameters are depicted in Figure 3. For comparison, similar results for the EBB beam are also plotted. It can be seen that the critical temperature of a metal-rich beam $(n=8)$ is lower than that of a geometrically identical ceramic-rich beam $(n=0)$. Because of the neglect of transverse shear deformations, the critical temperature for an EBB is higher than that of the corresponding TB. For the TB, an increase in the slenderness ratio results in higher values of the critical temperature because of the decrease in the influence of shear deformations with an increase in the slenderness ratio of the beam. As the slenderness ratio approaches infinity, the critical temperature of the TB tends to that of the EBB.

In Table 4 we have listed coordinates of the buckling mode transition points for a S-S homogeneous beam and compared them with those available in the literature for the pure metal beam. It is clear that the presently computed values of $K_{1}$ for the buckling mode transitions agree well with those of Ref. [24], wherein a mechanical problem has been studied.

\section{Analysis of post-buckling deformations}

When the thermal load $\tau$ exceeds the critical temperature $\tau_{c r}$, the FGM beam is in the post-buckled state. Thermally post-buckled equilibrium paths have been computed by the continuation method in which the parameter $m_{0}$ (or $\left.\varphi_{0}\right)$ is increased in small steps. Due to symmetry of beam's deformations about the

Table 2. Coordinates of transition points for the C-C FGM beam and various values of the exponent $n$ under uniform temperature rise $\left(T_{r}=1\right)$.

\begin{tabular}{lccccc}
\hline & & \multicolumn{3}{c}{$\left(K_{1} / \pi^{4}, \tau_{c r} / \pi^{2}\right)$} \\
\cline { 3 - 6 }$n$ & Source & $A_{01}$ & $A_{12}$ & $A_{23}$ & $A_{34}$ \\
\hline 0 & This work (TB) & $(0,9.126)$ & $(17.403,22.217)$ & $(125.888,43.499)$ & - \\
0.5 & This work (TB) & $(0,6.262)$ & $(12.442,15.028)$ & $(88.431,28.927)$ & $(287.617,46.729)$ \\
5 & This work (TB) & $(0,4.940)$ & $(10.24,11.848)$ & $(70.807,22.986)$ & $(233.193,37.216)$ \\
& & & & & \\
$\infty$ & This work (TB) & $(0,4.029)$ & $(8.055,9.622)$ & $(55.941,18.773)$ & $(196.43,31.169)$ \\
& This work (EBB) & $(0,4.069)$ & $(8.467,10.011)$ & $(62.169,20.087)$ & $(216.358,33.8797)$ \\
& Li and Batra [7] (EBB) & $(0,4)$ & $(9,10)$ & $(64,20)$ & $(225,34)$ \\
& Wu and Zhong [24] (EBB) & - & $(9,-)$ & $(64,-)$ & - \\
\hline
\end{tabular}

Table 3. Coordinates of transition points for C-C FGM beam for different values of the temperature rise ratio, $T_{r}(n=0.5)$.

\begin{tabular}{lcccc}
\hline & \multicolumn{4}{c}{$\left(K_{1} / \pi^{4}, \tau_{c r} / \pi^{2}\right)$} \\
\cline { 2 - 5 }$T_{r}$ & $A_{01}$ & $A_{12}$ & $A_{23}$ & $A_{34}$ \\
\hline 0.2 & $(0,7.579)$ & $(10.084,17.485)$ & $(68.664,32.792)$ & $(225.118,53.434)$ \\
0.5 & $(0,7.012)$ & $(11.118,16.425)$ & $(74.759,30.923)$ & $(242.571,49.651)$ \\
1 & $(0,6.262)$ & $(12.442,15.028)$ & $(88.431,28.927)$ & $(287.617,46.729)$ \\
1.5 & $(0,5.798)$ & $(14.402,14.061)$ & $(98.373,27.350)$ & $(313.698,43.680)$ \\
2 & $(0,5.248)$ & $(13.207,12.630)$ & $(88.366,24.187)$ & $(278.672,38.680)$ \\
\hline
\end{tabular}




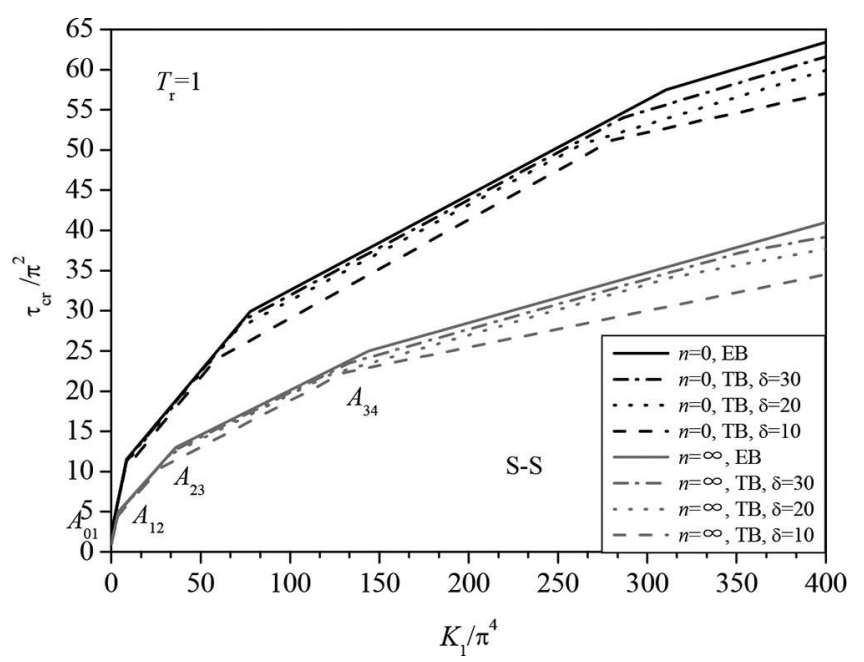

Figure 3. Critical temperature versus the linear foundation stiffness parameter for a S-S homogeneous beam under uniform temperature rise.

Table 4. Coordinates of transition points for a S-S homogeneous beam and uniform temperature rise $\left(T_{r}=1\right)$.

\begin{tabular}{|c|c|c|c|c|c|c|}
\hline \multirow[b]{2}{*}{$n$} & \multirow{2}{*}{\multicolumn{2}{|c|}{ Beam }} & \multicolumn{4}{|c|}{$\left(K_{1} / \pi^{4}, \tau_{c r} / \pi^{2}\right)$} \\
\hline & & & $A_{01}$ & $A_{12}$ & $A_{23}$ & $A_{34}$ \\
\hline \multirow[t]{4}{*}{0} & & EBB & $(0,2.300)$ & $(8.629,11.500)$ & $(77.668,29.901)$ & $(310.642,57.500)$ \\
\hline & TB & $\delta=30$ & $(0,2.292)$ & $(8.480,11.333)$ & $(74.331,28.890)$ & $(286.514,54.052)$ \\
\hline & & $\delta=20$ & $(0,2.282)$ & $(8.306,11.139)$ & $(70.789,27.809)$ & $(263.929,50.780)$ \\
\hline & & $\delta=10$ & $(0,2.231)$ & $(7.546,10.284)$ & $(58.176,23.923)$ & $(280.781,51.274)$ \\
\hline \multirow[t]{4}{*}{$\infty$} & & $\mathrm{EBB}$ & $(0,1)(0,1)^{*}$ & $(4,5)(4,5)^{*}(4,-)^{* * *}$ & $(36,13)(36,13) *(36,-)^{* *}$ & $(144,25)(144,25)^{*}(144,-)^{* *}$ \\
\hline & TB & $\delta=30$ & $(0,0.997)$ & $(3.931,4.927)$ & $(34.462,12.561)$ & $(132.850,23.504)$ \\
\hline & & $\delta=20$ & $(0,0.992)$ & $(3.850,4.843)$ & $(32.818,12.091)$ & $(122.322,22.076)$ \\
\hline & & $\delta=10$ & $(0,0.970)$ & $(3.498,4.471)$ & $(26.972,10.401)$ & $(130.190,22.295)$ \\
\hline
\end{tabular}

*Results from [7]; **Results from [24].

mid-section, the deflection of the beam centroid can also be regarded as the continuation parameter for an odd order buckling mode, such as the first, the third, and the fifth.

In Figures 4 and 5 we have exhibited post-buckled configurations of the first and the second modes of the C-C and the S-S FGM beam for different values of the thermal load $\tau$ and for $\left(K_{1}, K_{2}\right)=(500,0)$ and $(2000,50000)$. For each one of the four cases studied, the deflection of a point increases monotonically with an increase in the thermal load. We note that the C-C FGM beam with $n=5$ and $T_{r}=1$ buckles in mode 2 at $\tau=143.56$ for $\left(K_{1}, K_{2}\right)=(2000,50000)$ but in mode 1 for $\tau=94.77$ for $\left(K_{1}, K_{2}\right)=(500$, $0)$. Thus values of parameters of the elastic foundation noticeably affect the buckling temperature and the buckling mode. Similar remarks apply to the S-S FGM beam.

In Figure 6 we have plotted the relation between the centroidal deflection $W(0.5)$ and the thermal load $\tau$ for the C-C FGM beam for various values of the foundation stiffness and the power law exponent defining through-the-thickness variation of the material properties. The buckling mode corresponding to $K_{1}=10000$ is the third one whereas the beam buckles in the 1 st mode for $K_{1}=200,300,400$ and 500 . For a fixed buckling mode, the deflection decreases with an increase in the value of the linear foundation stiffness $K_{1}$. It is clear that the C-C FGM beam undergoes bifurcation-type buckling. With $K_{1}=300$ but $K_{2}$ varied from 100 to 150,000 , the beam buckles in mode 1 .

The non-linear foundation stiffness $K_{2}$ has a little influence on the buckling deformation when the thermal load is low and the influence becomes more obvious when the thermal load is larger, or the deformations become significant. For the same thermal load, beam's centroidal deflection decreases with an increase in the value of $K_{2}$. However, the centroidal deflection increases with an increase in 


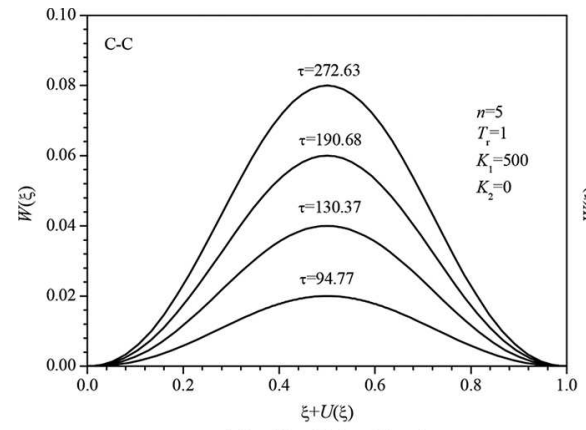

(a) $K_{1}=500, K_{2}=0$

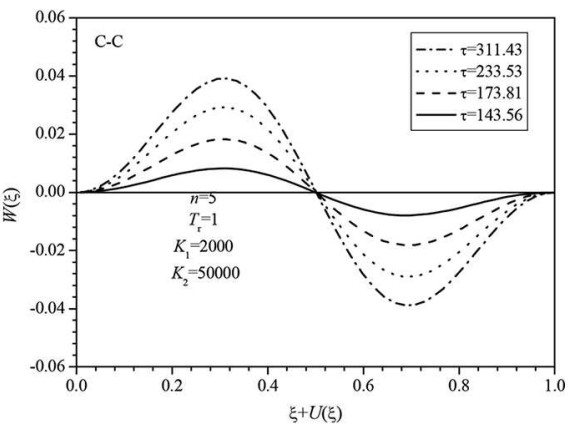

(b) $K_{1}=2000, K_{2}=50000$

Figure 4. The post-buckled configurations of a C-C FGM beam for $\left(K_{1}, K_{2}\right)=(500,0)$ and $(2000,50000)$.

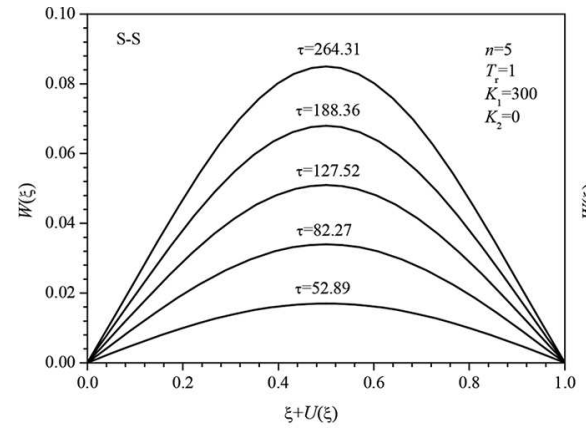

(a) $K_{1}=300, K_{2}=0$

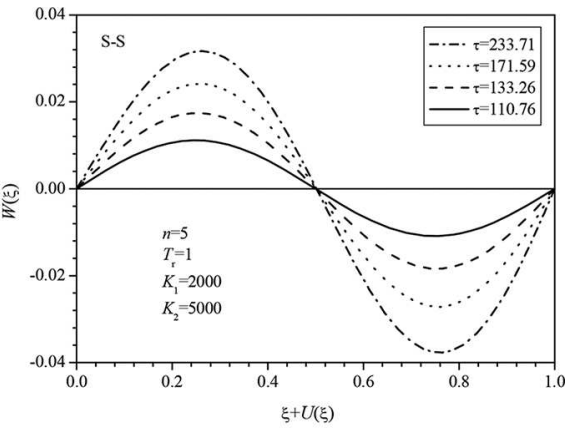

(b) $K_{1}=2000, K_{2}=50000$

Figure 5. The post-buckled configurations of a S-S FGM beam for $\left(K_{1}, K_{2}\right)=(300,0)$ and $(2000,50000)$.

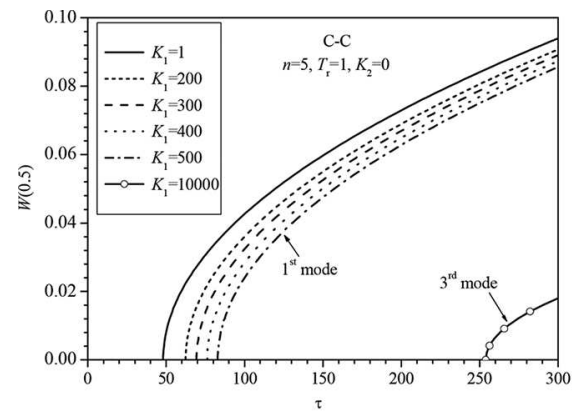

(a) For different values of $K_{1}$

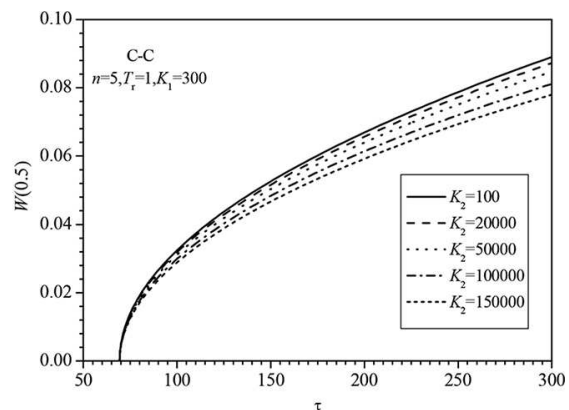

(b) For different values of $K_{2}$

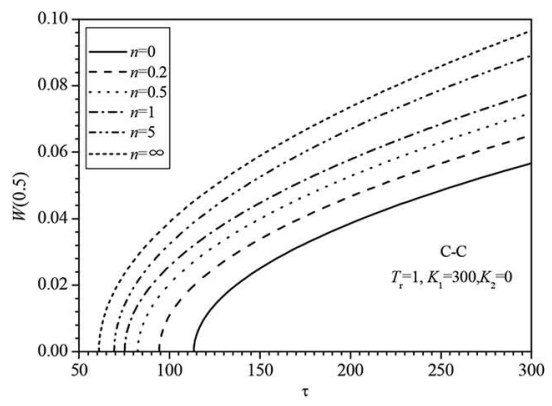

(c) For different values of $n$

Figure 6. For a C-C FGM beam the central deflection, $W(0.5)$, versus the thermal load $\tau$ for different values of (a) $K_{1}$, (b) $K_{2}$, and (c) the power law exponent, $n$. 
the thermal load and the lateral deflections of the third buckling mode are much smaller than those of the first mode for the same value of $\tau$. For $K_{1}=300, K_{2}=0$ and $T_{r}=1$, the C-C beam buckles in mode 1 for values of the power law exponent varying from 0 to $\infty$; the thermal buckling load is the least for the metallic beam $(n=\infty)$ and the most for the ceramic beam $(n=0)$.

For the S-S FGM beam we have depicted in Figure 7 the relationship between the centroidal deflection, $W(0.5)$, and the thermal load $\tau$ for different values of the foundation stiffness and the power law exponent. It is seen that only the equilibrium paths of a homogeneous beam under uniform temperature rise are of bifurcation-type. However, when either the beam is non-homogeneous or it is subjected to a non-uniform temperature rise, the equilibrium paths are similar to those of the homogeneous beam with an initial imperfection. The central deflection increases with an increase in the thermal load. Because deformations of the homogeneous beam under uniform temperature rise are symmetric about the beam mid-point, it can buckle either upwards or downwards depending upon values of the power law exponent and the temperature ratio. For a given value of the thermal load, a higher value of the linear foundation stiffness decreases the centroidal deflection of the beam. As for the C-C FGM beam, for a given thermal load centroidal deflections of the two homogeneous beams - one made of the ceramic and the other of the metal-bound centroidal deflections of the FGM beam. For $T_{r}=0.1$ and 0.5 , the beam deflects downwards but for $T_{r} \geq 1$ it deflects upwards.

In Figure 8 we have displayed the relationship between the end bending moment, $m(1)$, and the thermal load, $\tau$, for the C-C FGM beam for some values of the linear stiffness parameter, $K_{1}$, the volume fraction index, $n$, and the temperature ratio, $T_{r}$. For the unbuckled beam, we set $W \equiv \theta \equiv 0$ and $\Lambda_{0}=1$ in Eqs. (25)-(28) and (30), and get $m=\phi_{5} \tau / \delta=m_{T}$, which means that the bending moment is uniform and varies linearly with the thermal load as indicated by the straight line in Figure 8a. The dependence of the parameter $\phi_{5}$ upon the power law exponent $n$ is depicted in Figure 9 for a uniform temperature rise

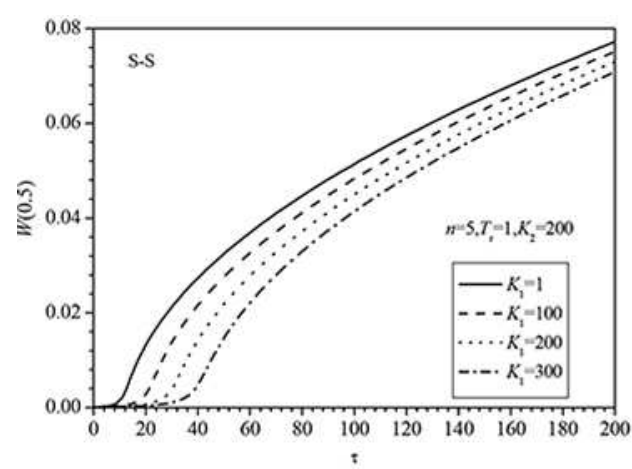

(a) For different values of $K_{1}$

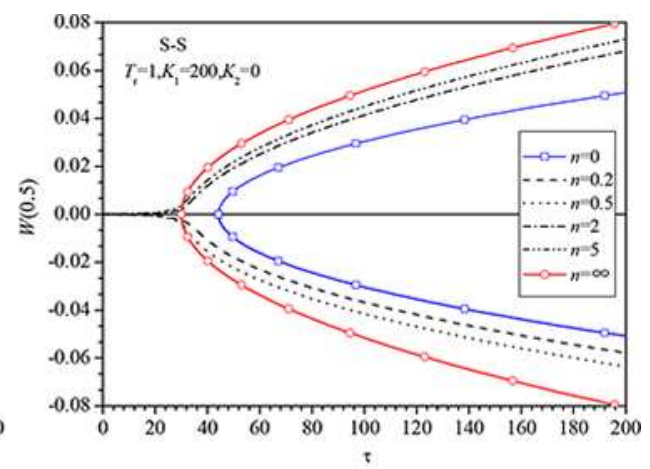

(b) For different values of $n$

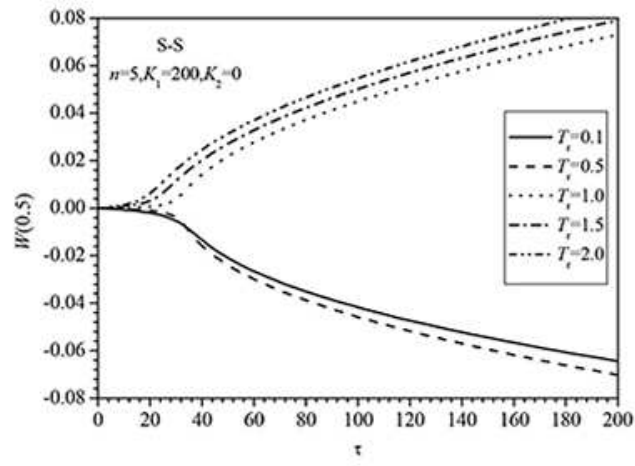

(c) For different values of $T$,

Figure 7. For a S-S FGM beam centroidal deflection, $W(0.5)$, versus the thermal load, $\tau$ for various values of parameters. 


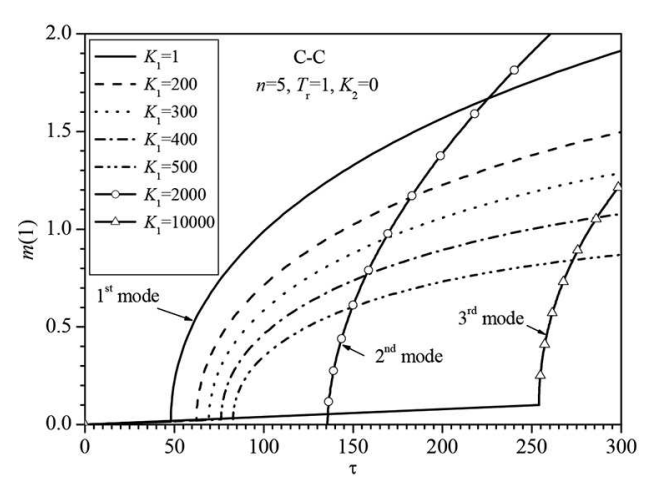

(a) For different values of $K_{1}$

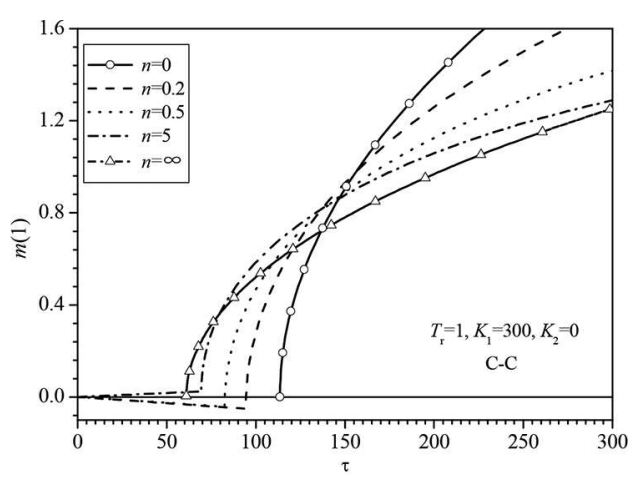

(b) For different values of $n$

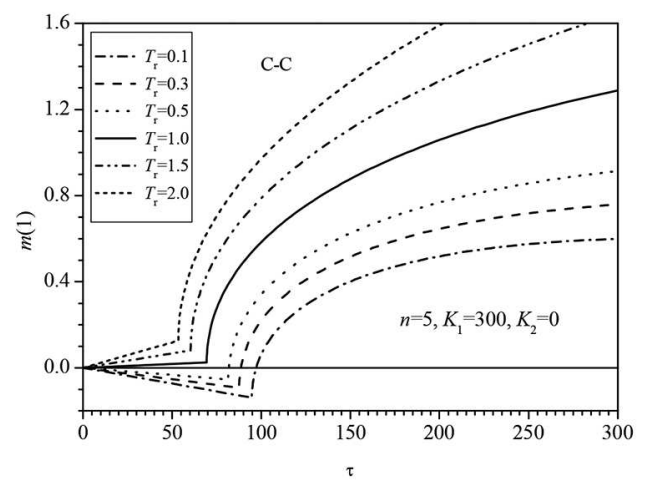

(c) For different values of $T_{r}$

Figure 8. For a C-C FGM beam, dependence of the end bending moment, $m(1)$, upon the thermal load, $\tau$, for different values of parameters.

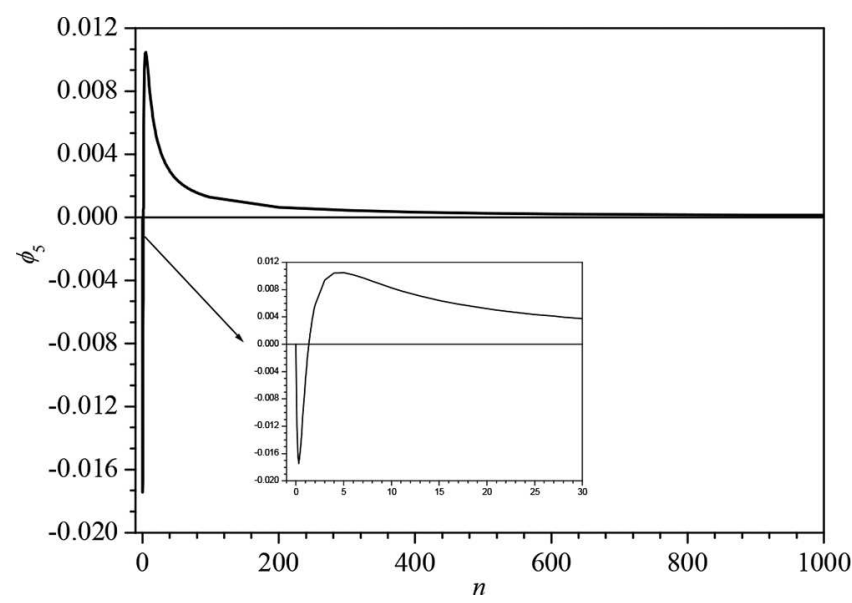

Figure 9. For a C-C FGM beam dependence of $\phi_{5}$ upon $n\left(T_{r}=1\right)$.

$\left(T_{r}=1\right)$. It can be seen that $\phi_{5}=0$ for the FGM beam with $n=0,1.349, \infty$ only; otherwise $\phi_{5} \neq 0$. For the post-buckled deformations in the first buckled mode and a uniform temperature rise, the end moment $m(1)$ decreases with an increase in the value of the linear foundation stiffness parameter $K_{1}$; cf. Figure 8a. 
By comparing results depicted in Figure $6 \mathrm{c}$ and Figure 8b, we see that even though the centroidal deflection monotonically decreases with an increase in the value of the power law exponent, $n$, the end moment $m(1)$ is not a monotonic function of $n$. The dependence of the end moment $m(1)$ upon the thermal load for different values of $T_{r}$ is exhibited in Figure $8 \mathrm{c}$. For a fixed value of the thermal load, the end bending moment monotonically increases with an increase in the value of $T_{r}$.

In Figure 10 we have displayed the characteristic curves of the horizontal resultant force, $P_{H}(1)$, versus the thermal load $\tau$ for the S-S FGM beam. For a homogeneous beam under uniform temperature rise, the resultant axial force prior to the onset of buckling is given by $P_{H}=\phi_{4} \tau=n_{T}$, which increases linearly with the thermal load and stays constant after buckling. For other values of the beam material and foundation stiffness parameters, there is no critical buckling temperature according to the traditional definition of buckling. Below a specified temperature that can be identified as a critical buckling temperature, the horizontal force also increases linearly with the thermal load. Above the specified temperature, the horizontal force changes slowly with an increase in the thermal load and in fact decreases with an increase in the thermal load for $n=0.2$ and 0.5 . Values of the linear stiffness of the foundation noticeably affect the temperature at which the slope of the $P_{H}(1)$ vs. the thermal load curve noticeably changes.

We show in Figure 11 the effect of the slenderness ratio on the beam centroidal deflection for S-S FGM EB and Timoshenko beams. It is clear that as the slenderness ratio increases, the difference between

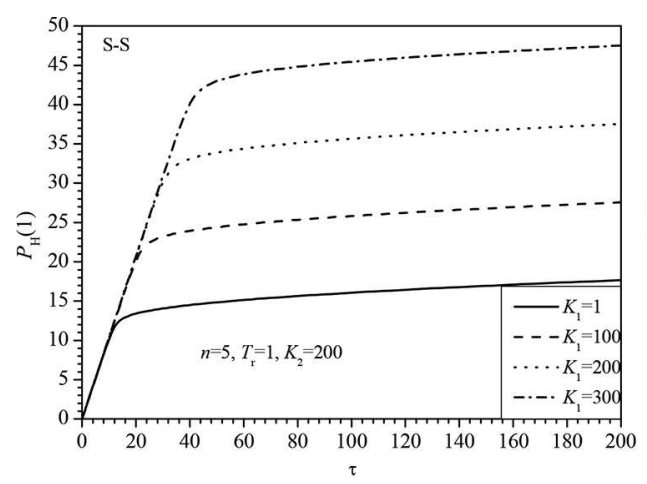

(a) For different values of $K_{1}$

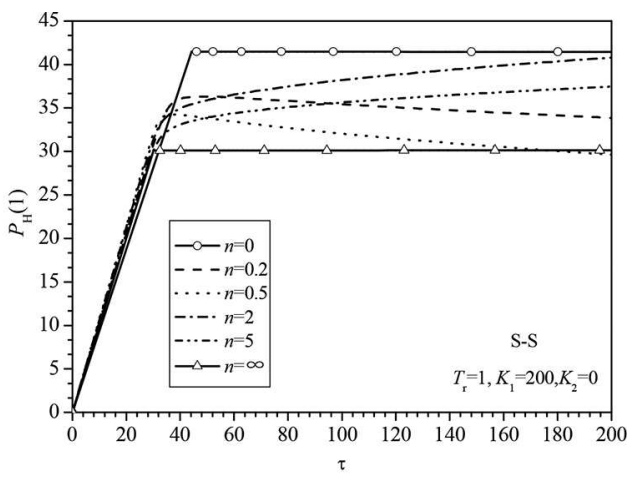

(b) For different values of $n$

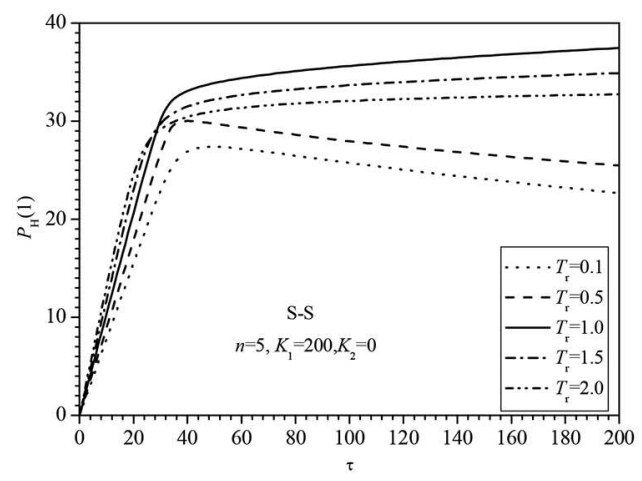

(c) For different values of $T_{r}$

Figure 10. For a S-S FGM beam, dependence of the horizontal force $P_{H}(1)$ upon the thermal load for different values of parameters. 


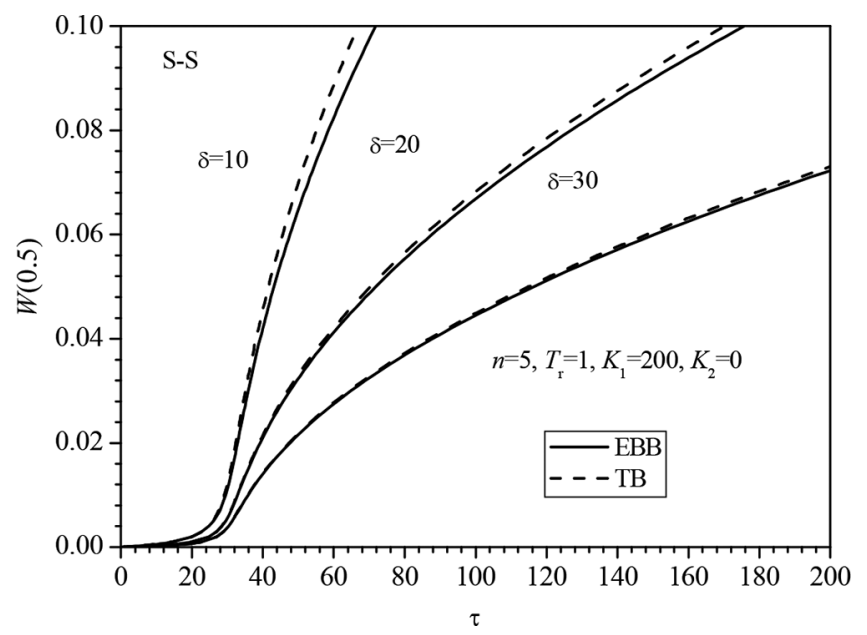

Figure 11. The effect of slenderness ratio on the central deflection of S-S FGM Euler-Bernoulli and Timoshenko beams.

deflections of the EBB and the TB becomes miniscule indicating the negligible effect of transverse shear deformations on the beam deflection for large values of the slenderness ratio.

\section{Conclusions}

Thermal buckling and post-buckling responses of a linear elastic FGM Timoshenko beam resting on a non-linear elastic foundation have been studied by solving one-way coupled equations for thermoelastic deformations of the beam. The non-linear strain-displacement relations that consider transverse shear strains have been used. The material properties are assumed to vary in the thickness direction according to a power law function of the thickness coordinate except that Poisson's ratio is assumed to be constant. For prescribed uniform temperatures on the top and the bottom surfaces of the beam, the through-the-thickness variation of the temperature is determined by solving the one-dimensional heat conduction equation. The coupled non-linear ordinary differential equations have been numerically solved by using the shooting method. Effects of various parameters upon thermally post-buckled equilibrium configurations have been illustrated. The following conclusions are drawn from the computed results.

Post-buckling behaviors of a C-C FGM Timoshenko beam exhibit bifurcations under both uniform and non-uniform temperature rise but those of a S-S beam do not. For a C-C FGM Timoshenko beam resting on a linear elastic foundation, the critical buckling modes exhibit transitions from the lower-one to the higher-one at well-defined values of the foundation stiffness and the thermal load.

For a C-C FGM Timoshenko beam with material properties given by a power law function of the thickness coordinate, the end bending moment need not have values between those for the homogeneous beams composed of materials of the bottom and the top surfaces of the FGM beam. For a S-S FGM Timoshenko beam the resultant axial force vs. the thermal load curve is bilinear with the point of intersection of the two linear parts representing the buckling load.

\section{Funding}

YS's and SRL's work was supported by the National Natural Science Foundation of China (11272278) and the China Postdoctoral Science Foundation funded project (149558). RCB's work was partially supported by the U.S. Office of Naval Research grant N00014-11-1-0594 with Dr. Rajapakse as the program manager. The authors gratefully acknowledge this support. 


\section{References}

[1] S. R. Li and C. J. Cheng, Analysis of Thermal Post-buckling of Heated Elastic Rods, Appl. Math. Mech., vol. 21, pp. $133-140,2000$.

[2] S. R. Li, Y. H. Zhou, and X. J. Zheng, Thermal Post-buckling of a Heated Elastic Rod with Pinned-Fixed Ends, J. Therm. Stresses, vol. 25, pp. 45-56, 2004.

[3] S. R. Li, C. J. Cheng, and Y. H. Zhou, Thermal Post-buckling of an Elastic Beam Subjected to a Transversely Nonuniform Temperature Rising, Appl. Math. Mech., vol. 24, pp. 514-520, 2003.

[4] S. R. Li and Y. H. Zhou, Geometrically Nonlinear Analysis of Timoshenko Beams under Thermomechanical Loadings, J. Therm. Stresses, vol. 26, pp. 861-872, 2003.

[5] S. R. Li and X. Song, Large Thermal Deflections of Timoshenko Beams under Transversely Non-uniform Temperature Rise, Mech. Res. Commun., vol. 33, pp. 84-92, 2006.

[6] X. Song and S. R. Li, Thermal Buckling and Post-buckling of Pinned-Fixed Euler-Bernoulli Beams on an Elastic Foundation, Mech. Res. Commun., vol. 34, pp. 164-171, 2007.

[7] S. R. Li and R. C. Batra, Thermal Buckling and Postbuckling of Euler-Bernoulli Beams Supported on Nonlinear Elastic Foundations, AIAA J., vol. 45, pp. 712-720, 2007.

[8] M. A. Vaz, M. S. Nascimento, and R. F. Solano, Initial Postbuckling of Elastic Rods Subjected to Thermal Loads and Resting on an Elastic Foundation, J. Therm. Stresses, vol. 30, pp. 381-393, 2007.

[9] Y. Kiani and M. R. Eslami, Thermal Buckling Analysis of Functionally Graded Material Beams, Inter. J. Mech. Mater. Design, vol. 6, pp. 229-238, 2010.

[10] Y. Kiani and M. R. Eslami, Thermomechanical Buckling of Temperature-dependent FGM Beams, Latin Amer. J. Solids Struct., vol. 10, pp. 223-245, 2013.

[11] L. S. Ma and D. W. Lee, Exact Solutions for Nonlinear Static Responses of aShear Deformable FGM Beam under an In-plane Thermal Loading, Euro. J. Mech. - A/Solids, vol. 31, pp. 13-20, 2012.

[12] F. Q. Zhao, Z. M. Wang, and H. Z. Liu, Thermal Post-buckling Analyses of Functionally Graded Material Rod, Appl. Math. Mech., vol. 28, pp. 59-67, 2007.

[13] S. R. Li, J. H. Zhang, and Y. G. Zhao, Thermal Post-buckling of Functionally Graded Material Timoshenko Beams, Appl. Math. Mech., vol. 27, pp. 803-810, 2006.

[14] L. S. Ma and D. W. Lee, A Further Discussion of Nonlinear Mechanical Behavior for FGM Beams under In-plane Thermal Loading, Compos. Struct., vol. 93, pp. 831-842, 2011.

[15] K. S. Anandrao, R. K. Gupta, P. Ramchandran, and G. V. Rao, Thermal Post-buckling Analysis of Uniform Slender Functionally Graded Material Beams, Structural Engineering and Mechanics, vol. 36, pp. 545-560, 2010.

[16] S. Sahraee and A. R. Saidi, Free Vibration and Buckling Analysis of Functionally Graded Deep Beam-columns on Two-parameter Elastic Foundations Using the Differential Quadrature Method, Proc. Inst. Mech. Eng., Pt. C: J. Mech. Eng. Sci., vol. 223, pp. 1273-1284, 2009.

[17] A. Fallah and M. M. Aghdam, Nonlinear Free Vibration and Post-buckling Analysis of Functionally Graded Beams on Nonlinear Elastic Foundation, Euro. J. Mech. - A/Solids, vol. 30, pp. 571-583, 2011.

[18] A. Fallah and M. M. Aghdam, Thermo-mechanical Buckling and Nonlinear Free Vibration Analysis of Functionally Graded Beams on Nonlinear Elastic Foundation, Compos. Pt. B: Engineering, vol. 43, pp. 1523-1530, 2012.

[19] S. E. Esfahani, Y. Kiani, and M. R. Eslami, Non-linear Thermal Stability Analysis of Temperature Dependent FGM Beams Supported on Non-linear Hardening Elastic Foundations, Inter. J. Mech. Sci., vol. 69, pp. 10-20, 2013.

[20] S. E. Ghiasian, Y. Kiani, and M. R. Eslami, Dynamic Buckling of Suddenly Heated or Compressed FGM Beams Resting on Nonlinear Elastic Foundation, Compos. Struct., vol. 106. pp. 225-234, 2013.

[21] S. E. Esfahani, Y. Kiani, M. Komijani, and M. R. Eslami, Vibration of a Temperature-dependent Thermally Pre/postbuckled FGM Beam over a Nonlinear Hardening Elastic Foundation. Journal of Applied Mechanics, vol. 81, pp. 011004, 2014.

[22] H. Shen and Z. Wang, Nonlinear Analysis of Shear Deformable FGM Beams Resting on Elastic Foundations in Thermal Environments, Inter. J. Mech. Sci., vol. 81, pp. 195-206, 2014.

[23] G. N. Praveen and J. N. Reddy, Nonlinear Transient Thermoelastic Analysis of Functionally Graded Ceramic-metal Plates, Inter. J. Solids Struct., vol. 35, pp. 4457-4476, 1998.

[24] B. S. Wu and H. X. Zhong, Postbuckling and Imperfection Sensitivity of Fixed-end and Free-end Struts on Elastic Foundation. Arch. Appl. Mech., vol. 69, pp. 491-498, 1999.

[25] R. C. Batra, Comparison of Results from Four Linear Constitutive Relations in Isotropic Finite Elasticity, Int. J. Nonlin. Mech., vol. 36, pp. 421-432, 2001.

[26] O. Allix and A. Corigliano, Geometrical and Interfacial Non-linearities in the Analysis of Delamination in Composites, Int. J. Solids Struct., vol. 36, pp. 2189-2216, 1999.

[27] R. C. Batra and J. Xiao, Finite Deformations of Full Sine-wave St.-Venant Beam Due to Tangential and Normal Distributed Loads using Nonlinear TSNDT, Meccanica, vol. 50, pp. 355-365, 2015. 